\title{
Boundary optimal control of time-space SIR model with nonlinear Robin boundary condition
}

\author{
E.-H. Essoufi ${ }^{1}$ - A. Zafrar $^{1}$ (D) \\ Received: 7 July 2021 / Revised: 11 October 2021 / Accepted: 11 October 2021 / Published online: 6 November 2021 \\ (c) The Author(s), under exclusive licence to Springer-Verlag GmbH Germany, part of Springer Nature 2021
}

\begin{abstract}
A boundary optimal control problem arising in time-space SIR epidemic models is treated. In this work we aim with the control of the flux of infected individuals crossing part of boundary. On the other side of the domain, we suppose a nonlinear boundary condition of third kind: nonlinear Robin boundary condition, this condition models immersing individual crossing this part of the boundary of the domain of study. We give the existence and uniqueness of the solution of both state and optimal control problem ending some numerical tests throughout a simple example.
\end{abstract}

Keywords Optimal control · Robin problem · SIR model

Mathematics Subject Classification 49J20 $\cdot$ 35J65 - 92B05

\section{Introduction and problem setting}

\subsection{Introduction}

Mathematical modelling have been extensively employed for obtaining interesting quantitative and qualitative information in different fields especially in the healthcare sector. These informations are the basic criterion to provide useful guidelines to outbreak management and policy development. Epidemiology is one of essential population biology disciplines that is concerned with public health. Epidemiological models provide important powerful means for predicting the spread of a disease and then controlling and understanding the dynamics which effect its transmission and diffusion through a given population. A famous example of such model based on the compartmental analysis is the Susceptible-Infected-Recovered (SIR) model and its variants, for epidemic [1-3], the Lotka-Volterra model for predator-prey dynamics $[1,4]$ and demographic and migration models established in sociology and demography $[5,6]$.

The majority of models confronted in such literature are based on compartmental analysis and consisting of systems

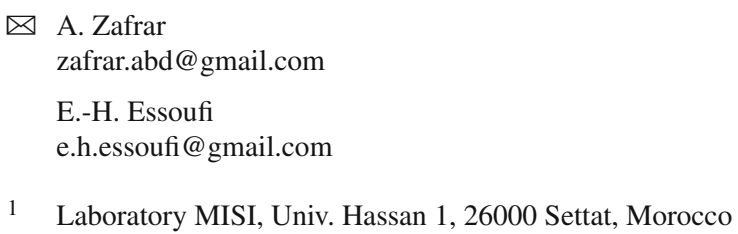

1 Laboratory MISI, Univ. Hassan 1, 26000 Settat, Morocco

of ordinary differential equations (ODEs). In spite that these models are simple in their formulation, analysis, and simple to solve mathematically and numerically, they are inadequate to describe diffusion and dynamic in both space and time. A common way to deal with introduce spatial variation into such ODE models is by considering meta-population models (models describing a population consisting of $n$ subpopulations which are connected by immigration or emigration) $[7,8]$, in other words by defining regional compartments corresponding to different areas in physical space, with coupling terms added to the model equations to account for the movement of species among the different regions [811]. This approach was lately developed in $[12,13]$ to model and describe the spread of the recent pandemic produced by COVID-19 virus among the different regions in Italy. Despite that this approach may be adequate for some application and description of complex spatial dynamics within compartments is difficult and possibly even non-feasible in this framework. Moreover, Some existing work consider an optimal control of SIR model by introducing density of vaccination in the model which is treated as control function [14-17] and then minimize a given cost function. This procedure can be considered as introducing a new compartment of vaccination and look for a density that decrease the number of infected and susceptible individuals in considered population.

In this paper we consider a compartmental SIR-model based on partial differential equations (PDEs) which include 
spatial information more naturally [18-23]. Generally, PDE models take into consideration a space continuous description of the suitable dynamics, enabling the description of dynamics in both time and space beyond all scales. This furnishes a significant interest over ODE models, whose capacity to describe spatial information is limited by the number of spatial compartments that the model includes. Our contribution consists in a boundary optimal control problem [24-27] subject to an SIR-diffusion model describing a time-space spread of a disease. The constraint is a system of three PDEs with Dirichlet and Neumann boundary condition concerning the $S$ and $R$ unknown. Concerning the third unknown, we adopt a non linear Robin boundary condition (boundary condition of third kind), this is motivated by the fact that the Robin boundary conditions describe when part of the infected individuals $I$ is "immersed" at the prescribed part of the boundary. This is the case where infected individuals can travel between different region and we interested in the influence of this hypothesis on the population of the specific region $\Omega$ subject to our study. On the second part of the boundary, we control the flux of individual constituting the population.

The present paper is Briefly outlined as follows. In the next section we introduce the time splitting method in order to handle the non linearity and we state the existence and uniqueness of the solution. In Sect. 3 we study the optimal control problem and provide a necessary optimality condition. Section 4 is devoted to the numerical illustrations by mean of simple example.

\subsection{Problem setting}

We start this subsection with some useful notations. Let $\Omega$ be an open bounded polyhedral domain subset of $\mathbb{R}^{d}(d=$ $1,2,3)$ with a boundary $\Gamma=\partial \Omega$. We define the norm $\|\cdot\|_{L^{2}(\Gamma)}$ for the Sobolev space $L^{2}(\Gamma)$. We shall denote by $\left(H^{1}(\Omega)\right)^{\prime}$ the dual space of the Sobolev space $H^{1}(\Omega)$, and similarly $\left(H^{\frac{1}{2}}(\Gamma)\right)^{\prime}$.

Let the boundary $\Gamma$ consist of three disjoint parts $\Gamma=$ $\Gamma_{1} \cup \Gamma_{2} \cup \Gamma_{3}$, with $\Gamma_{1}$ and $\Gamma_{2}$ being the union of some $(d-$ 1)-dimensional polyhedral domains. To consider a spatiallytemporally dependent problem, we define the spatial-temporal domains $Q$ and $\Sigma_{i}$, for $i=1,2,3$, respectively, by

$Q=(0, T) \times \Omega$ and $\Sigma_{i}=(0, T) \times \Gamma_{i}$

The thermal conductivity $q(x) \in C(\bar{\Omega})$ satisfies $0<q_{0} \leq$ $q \leq q_{1}<\infty$ for some positive constants $q_{0}$ and $q_{1}$. In the following sections, we shall frequently use the notation $C$ to denote a generic constant, which may differ at every situation. When no confusion can occur, we shall ignore the symbols $d x, d s$ and $d t$ in the integrals for notational simplicity.
Now we are in position to state our reaction-diffusion SIR model, which will be the direct problem with nonlinear Robin problem as follows:

$\begin{cases}\partial_{t} u-\nabla \cdot(q \nabla u)=f_{\beta, \gamma}(u), & \text { in } Q \\ q \frac{\partial u}{\partial n}+g(u)=0, & \text { on } \Sigma_{1} \\ q \frac{\partial u}{\partial n}=\sigma, & \text { on } \Sigma_{2} \\ u=0, & \text { on } \Sigma_{3} \\ u(x, 0)=u_{0}(x), & \end{cases}$

where $u=\left[\begin{array}{c}S \\ I \\ R\end{array}\right], f_{\beta, \gamma}(u)=\left[\begin{array}{c}-\beta S I \\ \beta S I-\gamma I \\ \gamma I\end{array}\right], g=$ $\left[\begin{array}{c}g_{1} \\ g_{2}(I) \\ g_{3}\end{array}\right], \quad q=\left[\begin{array}{c}q_{1} \\ q_{2} \\ q_{3}\end{array}\right], \sigma=\left[\begin{array}{c}0 \\ \sigma_{2} \\ 0\end{array}\right]$.

In order to afford the existence and uniqueness, we suppose that the map $g_{2}(\cdot, \cdot)$ satisfies the following properties

$$
g_{2}(x, 0)=0 \text { a.e. in } \Gamma_{1}
$$

$g_{2}(\cdot, \xi)$ is measurable a.e. in $\mathbb{R}$ $g_{2}(x, \cdot)$ is strictly increasing a.e. in $\Gamma_{1}$

$g_{2}(x, \cdot)$ is continuous a.e. in $\Gamma_{1}$

$$
\lim _{\xi \rightarrow \infty} g(x, \xi)=\infty \text { a.e. in } \Gamma_{1}
$$

After all $g(x, \cdot)$ is strictly increasing for a.e. $x \in \Gamma_{1}$, then it has an inverse which we express as $\widetilde{g}(x, \cdot)$. Let $G, \widetilde{G}$ : $\Gamma_{1} \times \mathbb{R} \rightarrow[0, \infty)$ be defined by

$G(x, u):=\int_{0}^{u} g(x, \eta)$ and $\widetilde{G}(x, u):=\int_{0}^{u} \widetilde{g}(x, \eta)$

It follows that for a.e. $x \in \Gamma_{1}, G(x, \cdot)$ and $\widetilde{G}(x, \cdot)$ are continuous, strictly increasing, convex and $G(x, 0)=\widetilde{G}(x, 0)=0$.

Definition 1.1 [28] The functions $G(x, \cdot)$ and $\widetilde{G}(x, \cdot)$ are called complementary $\mathcal{N}$-functions.

Definition 1.2 1. $\left(\triangle_{2}\right)$-condition: We say that $G$ and $\widetilde{G}$ satisfy the $\left(\triangle_{2}\right)$-condition, if there exist constants $0<$ $c_{1}, c_{2} \leq 1$ such that

$\left(\triangle_{2}\right)\left\{\begin{array}{l}c_{1} \xi g(x, \xi) \leq G(x, \xi) \leq \xi g(x, \xi), \\ c_{2} \xi \widetilde{g}(x, \xi) \leq \widetilde{G}(x, \xi) \leq \xi \widetilde{g}(x, \xi)\end{array}\right.$

for all $\xi \in \mathbb{R}$ and a.e. $x \in \Gamma_{1}$.

2. The condition $(\mathrm{G})$ : We say that $g$ satisfies the condition (G) if

(G) $\left\{\begin{array}{l}\text { there is a constant } c \in(0,1] \text { such that } \\ c|g(x, \xi-\eta)| \leq|g(x, \xi)-g(x, \eta)|\end{array}\right.$ 
for all $\xi, \eta \in \mathbb{R}$ and a.e. $x \in \Gamma_{1}$.

Let $\gamma u$ be the trace of $u$ on $\Gamma_{1}$ and let $g: \Gamma_{1} \times \mathbb{R} \rightarrow \mathbb{R}$ satisfy (1.2) and $G, \widetilde{G}: \Gamma_{1} \times \mathbb{R} \rightarrow[0, \infty)$ satisfy the $\left(\triangle_{2}\right)$ condition. We denote by $\mathbb{L}_{G}\left(\Gamma_{1}\right)$ the following MusielakOrlicz space

$\mathbb{L}_{G}\left(\Gamma_{1}\right):=\left\{u \in M\left(\Gamma_{1}\right): G(\cdot, u) \in L^{1}\left(\Gamma_{1}\right)\right\}$

where we denote by $M\left(\Gamma_{1}\right)$ is the space of all measurable functions on $\Gamma_{1}$. Since $G$ and $\widetilde{G}$ satisfy the $\left(\triangle_{2}\right)$-condition, it follows from [28] that $\mathbb{L}_{G}\left(\Gamma_{1}\right)$ endowed with the Luxemburg norm

$\|u\|_{G, \Gamma_{1}}:=\inf \left\{k>0:\|G(\cdot, u / k)\|_{L^{1}\left(\Gamma_{1}\right)} \leq 1\right\}$

is a reflexive Banach space. In addition, by [28] one has the following generalized version of Hölder's inequality:

$\left|\int_{\Gamma_{1}} u v\right| \leq 2\|u\|_{G, \Gamma_{1}}\|v\| \widetilde{G}, \Gamma_{1}$

Now we introduce the state vector space $V$ given by

$V:=\left\{u \in H^{1}(\Omega): \gamma u \in \mathbb{L}_{G}\left(\Gamma_{1}\right)\right.$ and $u=0$ on $\left.\Gamma_{3}\right\}$

endowed with the norm

$\|u\|_{V}:=\|u\|_{H^{1}(\Omega)}+\|u\|_{G, \Gamma_{1}}$.

\section{Time splitting and existence of solution}

The system of Eq. (1.1) are split into two systems of sub equations as follows, the nonlinear reaction equations

$$
\left\{\begin{array}{l}
\frac{1}{2} \partial_{t} u=f_{\beta, \gamma},(u) \text { in } Q \\
u(x, 0)=u_{0}(x)
\end{array}\right.
$$

which are used for the first half of the time step, and the linear diffusion equations

$$
\begin{cases}\frac{1}{2} \partial_{t} u-\nabla \cdot(q \nabla u)=0, & \text { in } Q \\ q^{\top} \frac{\partial u}{\partial n}+g(u)=0, & \text { on } \Sigma_{1} \\ q^{\top} \frac{\partial u}{\partial n}=\sigma, & \text { on } \Sigma_{2} \\ u=0, & \text { on } \Sigma_{3}\end{cases}
$$

which are used for the second half of the time step. The numerical method used to solve the equations is the forward Euler scheme.
The above equations transform to

$u^{n+\frac{1}{2}}=u^{n}+2 \delta t f_{\beta, \gamma}^{n}$, in $\Omega$

where $f_{\beta, \gamma}^{n}=\left[\begin{array}{c}-\beta S^{n} I^{n} \\ \beta S^{n} I^{n}-\gamma I^{n} \\ \gamma I^{n}\end{array}\right], S^{n}, I^{n}$ and $R^{n}$ indicate the approximate values of $S, I$ and $R$ at position $x$ and time $n \delta t, n=0,1, \ldots$ and where $S^{n+\frac{1}{2}}, I^{n+\frac{1}{2}}$, and $R^{n+\frac{1}{2}}$ indicate the representative values at the half-time step.

Similarly, for the second half time step

$$
\begin{cases}u^{n+1}-2 \delta t \nabla \cdot\left(q \nabla u^{n+1}\right)=u^{n+\frac{1}{2}}, & \text { in } \Omega \\ q^{\top} \frac{\partial u^{n+1}}{\partial n}+g\left(u^{n+1}\right)=0, & \text { on } \Sigma_{1} \\ q \frac{\partial u^{n+1}}{\partial n}=\sigma, & \text { on } \Sigma_{2} \\ u^{n+1}=0, & \text { on } \Sigma_{3}\end{cases}
$$

where $g\left(u^{n+1}\right)=\left[\begin{array}{c}g_{1} \\ g_{2}\left(I^{n+1}\right) \\ g_{3}\end{array}\right]$.

Below our analysis will be reserved to the nonlinear elliptic boundary value problem given by (2.4). For the sake of simplicity we shall omit the exponent $n+1$ since the interval time is discretised we omit the dependence in time for the maps $g, \widetilde{g}, G$ and $\widetilde{G}$.

The weak formulation of (2.4) is give as follows

$$
\begin{gathered}
\int_{\Omega} u v+2 \delta t \int_{\Omega} q \nabla u \nabla v+2 \delta t \int_{\Gamma_{1}} g(u) v \\
=\int_{\Omega} u^{n+\frac{1}{2}} v+2 \delta t \int_{\Gamma_{2}} \sigma v
\end{gathered}
$$

Now, let us define the following nonlinear form

$a(u, v)=\int_{\Omega} u v+2 \delta t \int_{\Omega} q \nabla u \nabla v+2 \delta t \int_{\Gamma_{1}} g(u) v$

and we have the following result

Lemma 2.1 Assume that $g$ satisfies all assertions in (1.2) and that $\mathrm{G}$ and $\widetilde{G}$ satisfy the $\left(\triangle_{2}\right)$-condition. Then the bilinear form $a(\cdot, \cdot)$ is monotone and hemicontinuous and $a(u, \cdot) \in V^{\prime}$ for all $u \in V$. Furthermore $a(\cdot, \cdot)$ is coercive, i.e. $\frac{a(u, u)}{\|u\|_{V}} \rightarrow \infty$ as $\|u\|_{V} \rightarrow \infty$.

Proof Since $g(\cdot)$ is strictly increasing we have $a(u, u-v)-$ $a(v, u-u) \geq 0$ for $u, v \in V$, that is $a(\cdot, \cdot)$ is monotone. by the continuity argument of $g(\cdot)$ for a.e. $x \in \Gamma_{1}$, we obtain that for all $u, v, w \in V$,

$a(u+s v, w) \longrightarrow a(u, w)$ as $s \searrow 0$.

therefore $a(\cdot, \cdot)$ is hemicontinuous. 
Let us show that $a(u, \cdot) \in V^{\prime} \forall u \in V$. It is clear that $a(u, \cdot)$ is linear. Let $u, v \in V$. Using (1.3) we obtain that

$$
\begin{aligned}
|a(u, v)| \leq & \|u\|_{L 2}\|v\|_{L^{2}}+\|\nabla u\|_{L^{2}}\|\nabla v\|_{L^{2}}+\left|\int_{\Gamma_{1}} g(u) v d \Gamma\right| \\
\leq & C\|u\|_{V}\|v\|_{V} \\
& +2 \max \left\{1, \int_{\Gamma_{1}} \widetilde{G}(x, g(u)) d \Gamma\right\}\|v\|_{G, \Gamma_{1}} \\
\leq & \left(C\|u\|_{V}+2 \max \left\{1, \int_{\Gamma_{1}} \widetilde{G}(x, g(u)) d \Gamma\right\}\right)\|v\|_{V}
\end{aligned}
$$

Using the fact that $0 \leq \widetilde{G}(x, g(\xi)) \leq\left(1 / c_{1}\right) G(x, \xi)$, we get that $a(u, \cdot) \in V^{\prime}$ for every $u \in V$. Finally, because of both $G$ and $\widetilde{G}$ satisfy the $\left(\triangle_{2}\right)$-condition, it follows that

$\frac{\int_{\Gamma_{1}} g(u) u d \sigma}{\|u\|_{G, \Gamma_{1}}} \longrightarrow+\infty$ as $\|u\|_{G, \Gamma_{1}} \rightarrow+\infty$

we get immediately

$\lim _{\|u\|_{V} \rightarrow+\infty} \frac{a(u, u)}{\|u\|_{V}}=+\infty$

as consequence $a(\cdot, \cdot)$ is coercive.

The existence and uniqueness of the solution of the problem (2.4) is stated in the following

Theorem 2.2 Assume that $g$ satisfies (1.2) and that $G$ and $\widetilde{G}$ satisfy the $\left(\triangle_{2}\right)$-condition. Let $f \in L^{p}(\Omega)$ then the problem (2.4) has a unique weak solution. Moreover we have the following majoration

$\|u\|_{H^{1}} \leq C\left(\left\|f_{\beta, \alpha}\right\|_{L^{p}}+\|\sigma\|_{L^{2}\left(\Gamma_{2}\right)}\right)$

In the remainder part of this paper we restrict ourself to the $\mathcal{N}$-functions where $g(u)$ given by

$G(u)=e^{u}-u-1$

Then the function $g(\cdot)$ obtained from $G(\cdot)$ have some interesting properties:

(1) For a nonnegative integer $k, g(\cdot)$ is $k$-differentiable;

(2) For $u \in H^{1}(\Omega), g(u) \in L^{2}(\Gamma)$;

(3) For $u \in H^{1}(\Omega), g(|u|) \in L^{1}(\Gamma)$ and there exists a positive constant $\kappa$ such that

$$
\begin{aligned}
& \int_{\Gamma} g(|u|) \leq 1+\operatorname{meas}(\Gamma) \\
& \quad+\operatorname{meas}(\Gamma) e^{\kappa^{2}\|u\|_{V}^{2}}+\|u\|_{V}^{2}<\infty,
\end{aligned}
$$

where meas $(\Gamma)$ is the measure of $\Gamma$.
In fact (see [29]), let $u$ be given in $H^{1}(\Omega)$. Then $u \in H^{1 / 2}(\Gamma)$. Using embedding results for Orlicz-Sobolev spaces (see [28]) since $\Omega \subset \mathbb{R}^{2}$, we have $H^{1 / 2}(\Gamma) \subset \subset L_{\mathrm{G}}$ where the $\mathcal{N}$ function $G(u)=\mathrm{e}^{u^{2}}-1$. Thus there exists a constant $\kappa>0$ such that

$\|u\|_{L_{\mathrm{G}}} \equiv \inf \left\{\lambda ; \int_{\Gamma} \mathrm{e}^{\frac{|u|^{2}}{\lambda^{2}}}-1 \leq 1\right\} \leq \kappa\|u\|_{V}<\infty$

Hence for a small (enough) $\epsilon>0$ and for $r=\|u\|_{\mathbb{L}_{G}}+\epsilon$ we obtain

$\int_{\Gamma}\left[\mathrm{e}^{\frac{|u|^{2}}{r^{2}}}-1\right] \leq 1$

so that

$\int_{\Gamma} \mathrm{e}^{\frac{|u|^{2}}{r^{2}}} \leq 1+\operatorname{meas}(\Gamma)$

Let us set $M=r^{2}$. An elementary calculation gives

$\mathrm{e}^{x}<\mathrm{e}^{x^{2}} \quad \forall|x|>M$

Setting $K=\mathrm{e}^{M}$, i.e., $K=\mathrm{e}^{r^{2}}<\infty$, we obtain

$$
\begin{aligned}
\int_{\Gamma} \mathrm{e}^{|u|} & =\int_{\{\mathbf{x} \in \Gamma:|u(\mathbf{x})| \geq M\}} \mathrm{e}^{|u|}+\int_{\{\mathbf{x} \in \Gamma:|u(\mathbf{x})|<M\}} \mathrm{e}^{|u|} \\
& \leq \int_{\Gamma} \mathrm{e}^{|u|^{2}} d \Gamma+K \operatorname{meas}(\Gamma) \\
& \leq 1+\operatorname{meas}(\Gamma)+\operatorname{meas}(\Gamma) \mathrm{e}^{\left(\|u\|_{L_{\mathrm{A}}}+\epsilon\right)^{2}} \\
& \leq 1+\operatorname{meas}(\Gamma)+\operatorname{meas}(\Gamma) \mathrm{e}^{\left(\kappa\|u\|_{V}+\epsilon\right)^{2}} \quad<\infty .
\end{aligned}
$$

making $\epsilon \rightarrow 0$ achieves the argument.

Next our contribution is concerned in the formulation of optimal control problem. The classic way to do this is as follows: Given a measurement or observation data $u_{o b}$ of $u$, find an optimal control $\sigma$ such that the desired profile is reached.

The regularized problems of optimal control problem then given as the following minimization problems:

$$
\min _{(\sigma, u) \in \mathcal{C} \times K} J(\sigma, u):=\frac{1}{2} \int_{\Omega}\left|u(\sigma)-u_{o b}\right|^{2}+\varepsilon \mathcal{R}(\sigma)
$$

subject to the parabolic equation (2.4), where $\mathcal{C}$ is a subset of $L^{2}(\Omega)$ defined by

$\mathcal{C}=\left\{\sigma \in L^{2}\left(\Gamma_{2}\right) ; / \underline{\sigma} \leq \sigma \leq \bar{\sigma}\right\}$

$\varepsilon>0$ is a regularisation parameter.

We have the following result due to [29]: 
Lemma 2.3 Suppose that $\left\{u_{k}\right\} \subset L^{2}\left(\Gamma_{1}\right)$ is a sequence such that $u_{k} \rightarrow u$ a.e. on $\Gamma_{1}, g$ satisfies (1.2) and

$\int_{\Gamma_{1}} g\left(u_{k}\right) u_{k} d \Gamma \leq C \quad \forall k$

where $C>0$ is a constant independent of $k$. Then

$\int_{\Gamma_{1}} g(u) u d \Gamma \leq \liminf _{k \rightarrow \infty} \int_{\Gamma_{1}} g\left(u_{k}\right) u_{k} d \Gamma$

and

$\lim _{k \rightarrow \infty} \int_{\Gamma_{1}}\left|g\left(u_{k}\right)-g(u)\right| d \Gamma=0$

Proof By (1.2), $g$ is continuous and $u_{k} \rightarrow u$ a.e. on $\Gamma_{1}$, we deduce that $g\left(u_{k}\right) \rightarrow g(u)$ a.e. on $\Gamma_{1}$. Since $g$ is strictly increasing map we have $g(u) u \geq 0$ on $\Gamma_{1}$ so that we may use Fatou's result to get

$\int_{\Gamma_{1}} g(u) u d \Gamma \leq \liminf _{k \rightarrow \infty} \int_{\Gamma_{1}} g\left(u_{k}\right) u_{k} d \Gamma \leq C$

Hence $g(u) u \in L^{1}\left(\Gamma_{1}\right)$. Put $S=\sup _{|x| \leq 1}|g(x)|$, from the identity

$|g(t)|=|x|^{-1} g(x) x \quad \forall x \neq 0$

we conclude that

$|g(x)| \leq g(x) x+S \quad \forall x \in \mathbb{R}$

Thus

$|g(u)| \leq|g(u)||u|+S \quad$ on $\Gamma_{1}$

i.e., $g(u) \in L^{1}\left(\Gamma_{1}\right)$. Employing again the identity (2.12) we obtain, for each $\delta>0$ and for a.e. $\mathbf{x} \in \Gamma_{1}$, either

$\left|u_{k}\right| \leq \delta^{-1}$

or

$|g(u)| \leq \delta g\left(u_{k}\right) u_{k}$

so that

$|g(u)| \leq C_{\delta}+\delta g\left(u_{k}\right) u_{k} \quad$ on $\Gamma_{1}$

where $C_{\delta}=\sup _{|x| \leq \delta^{-1}}|g(x)|$. For every measurable subset $\Gamma_{s} \subset \Gamma_{1}$ we have

$\int_{\Gamma_{1}}\left|g\left(u_{k}\right)\right| d \Gamma \leq C_{\delta} \operatorname{meas}\left(\Gamma_{s}\right)+\delta \int_{\Gamma_{1}} g\left(u_{k}\right) u_{k} d \Gamma$
It is straightforward that

$\int_{\Gamma_{s}} g\left(u_{k}\right) u_{k} d \Gamma \leq C$

for $k$ greater than some given integer $N>0$. Thus

$\int_{\Gamma_{s}}\left|g\left(u_{k}\right)\right| d \Gamma \leq C_{\delta} \operatorname{meas}\left(\Gamma_{s}\right)+\delta C \quad \forall n>N$

where meas $\left(\Gamma_{s}\right)$ is the measure of $\Gamma_{s}$. Hence, the sequence of functions $\left\{g\left(u_{k}\right)\right\}$ has equi-absolutely continuous integrals. By Vitali's Convergence Theorem,

$\lim _{k \rightarrow \infty} \int_{\Gamma_{1}}\left|g\left(u_{k}\right)-g(u)\right| d \Gamma=0$.

We are now prepared to prove the existence of an optimal solution.

Theorem 2.4 Assume that $g(u) \in L^{2}\left(\Gamma_{1}\right)$ for $u \in H^{1}(\Omega)$. Then there exists an element $(\hat{u}, \hat{\sigma}) \in \mathcal{C} \times K$ that minimizes (2.10) subject to (2.4).

Proof Theorem 2.2 implies an element $(u, \sigma)$ exists such that $J(u, \sigma)<\infty$. Thus we may choose a minimizing sequence $\left\{\left(u_{k}, \sigma_{k}\right)\right\}$ such that

$\lim _{k \rightarrow \infty} J\left(u_{k}, \sigma_{k}\right)=\inf _{(u, \sigma) \in \mathcal{C} \times K} J(u, \sigma)$

and

$$
\begin{gathered}
\int_{\Omega} u_{k} v+2 \delta t \int_{\Omega} q \nabla u_{k} \nabla v+2 \delta t \int_{\Gamma_{1}} g\left(u_{k}\right) v \\
=\int_{\Omega} u^{n+\frac{1}{2}} v+2 \delta t \int_{\Gamma_{2}} \sigma_{k} v, \quad \forall v \in H^{1}(\Omega)
\end{gathered}
$$

We deduce that $\left\{\sigma_{k}\right\}$ is bounded in $L^{2}\left(\Gamma_{2}\right)$ and $\left\{\left\|u_{k}\right\|_{H^{1}}\right\}$ is bounded. Hence we can extract a subsequence $\left\{\left(u_{k}, \sigma_{k}\right)\right\}$ such that

$u_{k} \rightarrow \hat{u} \quad$ in $H^{1}(\Omega)$

and

$\sigma_{k} \rightarrow \hat{\sigma} \quad$ in $L^{2}\left(\Gamma_{2}\right)$.

Furthermore, trace theorems and compact embedding results imply $u_{k} \rightarrow u$ in $L^{2}\left(\Gamma_{1}\right)$; this in turn implies $u_{k} \rightarrow u$ pointwise a.e. on $\Gamma_{1}$ after extracting subsequence. By setting $v=u_{k}$ in (2.13) we obtain

$$
\int_{\Omega}\left|u_{k}\right|^{2}+2 \delta t \int_{\Omega} q\left|\nabla u_{k}\right|^{2}+2 \delta t \int_{\Gamma_{1}} g\left(u_{k}\right) u_{k}
$$




$$
\leq 2 \delta t\left\|\sigma_{k}\right\|_{0, \Gamma_{2}}\left\|u_{k}\right\|_{0, \Gamma_{1}} \leq C\left\|\sigma_{k}\right\|_{0, \Gamma_{2}}\left\|u_{k}\right\|_{H^{1}}
$$

Hence we deduce

$$
\int_{\Gamma_{1}} g\left(u_{k}\right) u_{k} \leq M
$$

where $M$ is a constant independent of $k$. By Lemma 2.3,

$$
\int_{\Gamma_{1}} g(\hat{u}) \hat{u} d \Gamma \leq \liminf _{k \rightarrow \infty} \int_{\Gamma_{1}} g\left(u_{k}\right) u_{k} d \Gamma
$$

and

$$
\lim _{k \rightarrow \infty} \int_{\Gamma_{1}}\left|g\left(u_{k}\right)-g(\hat{u})\right|=0
$$

For each $v \in C^{\infty}(\Omega),(2.14)$ allows us to pass to the limit in (2.13) to obtain

$$
\begin{aligned}
& \int_{\Omega} \hat{u} v+2 \delta t \int_{\Omega} q \nabla \hat{u} \nabla v+2 \delta t \int_{\Gamma_{1}} g(\hat{u}) v=\int_{\Omega} u^{n+\frac{1}{2}} v \\
& \quad+2 \delta t \int_{\Gamma_{2}} \hat{\sigma} v \quad \forall v \in C^{\infty}(\bar{\Omega})
\end{aligned}
$$

Then using the denseness of $C^{\infty}(\Omega)$ in $H^{1}(\Omega)$ and the fact that $g(\hat{u}) \in L^{2}\left(\Gamma_{1}\right)$, we obtain

$$
\begin{aligned}
& \int_{\Omega} \hat{u} v+2 \delta t \int_{\Omega} q \nabla \hat{u} \nabla v+2 \delta t \int_{\Gamma_{1}} g(\hat{u}) v=\int_{\Omega} u^{n+\frac{1}{2}} v \\
& \quad+2 \delta t \int_{\Gamma_{2}} \hat{\sigma} v \quad \forall \psi \in H^{1}(\Omega) .
\end{aligned}
$$

Thus $(\hat{u}, \hat{\sigma}) \in \mathcal{C} \times K$. Finally using the weak lower semicontinuity of $J(\cdot, \cdot)$, we conclude that $(\hat{u}, \hat{\sigma})$ is indeed an optimal solution, i.e.

$$
J(\hat{u}, \hat{\sigma})=\inf _{(u, \sigma) \in \mathcal{C} \times K} J(u, \sigma)
$$

\section{A necessary condition of optimality}

In this section we will derive a necessary condition that an optimal solution must satisfy. The existence of an optimal solution has been established in the above section. In Sect. 2 we have shown that for each $\sigma \in L^{2}\left(\Gamma_{2}\right)$, there exists a unique $u$ satisfying (2.5). Thus the state $u$ is a well-defined function of $\sigma$ and will be denoted by $u=u(\sigma)$.

Our aim is to state the necessary condition rigorously and express it in a more practical form. To this end we shall use the following theorem (see [30, Lemma 3.11, p. 1127] and [24])
Theorem 3.1 Let $\mathcal{B}$ be a Banach space $X$ and $Y$ two reflexive Banach spaces. Let also be two $\mathcal{C}^{1}$ functions

$F: \mathcal{B} \times X \longrightarrow Y, \quad L: \mathcal{B} \times X \longrightarrow \mathbb{R}$.

We suppose that, for all $\sigma \in \mathcal{B}$,

1. There exists a unique $u(\sigma)$ such that $F(\sigma, u(\sigma))=0$,

2. $\partial_{2} F(\sigma, u(\sigma))$ is an isomorphism from $X$ onto $Y$,

Then, $J(\sigma)=L(\sigma, u(\sigma))$ is differentiable and, for every $\zeta \in \mathcal{B}$,

$\frac{d J}{d \sigma}(\sigma) \zeta=\partial_{1} L(\sigma, u(\sigma)) \zeta-\left\langle p(\sigma), \partial_{1} F(\sigma, u(\sigma)) \zeta\right\rangle_{Y^{\prime}, Y}$

where $p(\sigma) \in Y^{\prime}$ is the adjoint state, unique solution to

$\left[\partial_{2} F(\sigma, u(\sigma))\right]^{*} p(\sigma)=\partial_{2} L(\sigma, u(\sigma))$ in $X^{\prime}$.

In order to apply this result we shall need the following preparations. By Riesz's representation theorem we define

$$
\begin{array}{r}
\langle\mathbf{y}(\sigma), v\rangle_{L^{2}\left(\Gamma_{2}\right)}=2 \delta t \int_{\Gamma_{2}} \sigma v \\
\langle G(u), v\rangle_{L^{2}\left(\Gamma_{1}\right)}=2 \delta t \int_{\Gamma_{1}} g(u) v \\
\langle A u, v\rangle=\int_{\Omega} u v+2 \delta t q \nabla u \nabla v \\
\left\langle\mathbf{u}^{n+\frac{1}{2}}, v\right\rangle=\int_{\Omega} u^{n+\frac{1}{2}} v,
\end{array}
$$

then we put

$F(\sigma, u)=A u+\mathbf{G}(u)-\mathbf{y}(\sigma)-\mathbf{u}^{n+\frac{1}{2}}$

Thus

$\partial_{1} F(\sigma, u) \xi=-\mathbf{y}(\xi)$

and

$\partial_{2} F(\sigma, u) w=A w+\mathbf{G}^{\prime}(u) w$

where

$\left\langle\mathbf{G}^{\prime}(u) w, v\right\rangle_{L^{2}\left(\Gamma_{1}\right)}=2 \delta t \int_{\Gamma_{1}} g^{\prime}(u) w v$.

Theorem 3.2 The first order necessary optimality condition reads: any optimal control $\sigma$ satisfies

$\sigma=-\frac{1}{\beta} p, \quad$ on $\Gamma_{2}$. 
where $p$ is the unique solution of

$\left\langle u-u_{o b}, w\right\rangle=\left\langle p, A w+\boldsymbol{G}^{\prime}(u) w\right\rangle$

Proof Let us put $\mathcal{B}=L^{2}\left(\Gamma_{2}\right), X=V$ and $Y=X^{\prime}$.

The first assertion is straightforward. The second assertion of Theorem 3.1 is fulfilled since the operator $A$ in $\partial_{2} F$ is coercive. Then, $J(\sigma)=L(\sigma, u(\sigma))$ is differentiable and, for every $\zeta \in \mathcal{B}$, which gives the condition (3.6).

\section{Finite element approximation and numerical example}

We start by introducing the optimality system formed by state variational equation, adjoint variational equation and optimality condition (3.6) as follows:

$$
\left\{\begin{array}{l}
\int_{\Omega} u v+2 \delta t \int_{\Omega} q \nabla u \nabla v+2 \delta t \int_{\Gamma_{1}} g(u) v=-\frac{2 \delta t}{\beta} \int_{\Gamma_{2}} p v+\int_{\Omega} u^{n+\frac{1}{2}} v, \quad \forall v \in V \\
\int_{\Omega} p \omega+2 \delta t \int_{\Omega} q \nabla p \nabla \omega+2 \delta t \int_{\Gamma_{1}} g^{\prime}(u) p \omega=2 \delta t \int_{\Omega}\left(u-u_{o b}\right) \omega, \quad \forall \omega \in V \\
\sigma=-\frac{1}{\beta} p, \quad \text { on } \Gamma_{2}
\end{array}\right.
$$

$$
\frac{d J}{d \sigma}(\sigma) \zeta=\partial_{1} L(\sigma, u(\sigma)) \zeta-\left\langle p, \partial_{1} F(\sigma, u(\sigma)) \zeta\right\rangle_{Y^{\prime}, Y}
$$

where $p(\sigma) \in Y^{\prime}$ is the adjoint state, unique solution to

$\left[\partial_{2} F(\sigma, u(\sigma))\right]^{*} p=\partial_{2} L(\sigma, u(\sigma))$ in $X^{\prime}$.

which is equivalent to

$\left\langle\left[\partial_{2} F(\sigma, u(\sigma))\right]^{*} p, w\right\rangle=\left\langle\partial_{2} L(\sigma, u(\sigma)), w\right\rangle$.

thus

$\left\langle p, \partial_{2} F(\sigma, u(\sigma)) w\right\rangle=\left\langle\partial_{2} L(\sigma, u(\sigma)), w\right\rangle$.

and this is exactly (3.7).

Now the necessary condition is given by

$\frac{d J}{d \sigma}(\sigma) \zeta=0, \quad$ for all $\xi$

and we have
A finite element discretization of the optimality system (4.1) is defined in the usual approach. We assume $\Omega$ is a convex polygonal domain and we choose families of finite dimensional subspaces $V^{h} \subset H^{1}(\Omega)$ satisfying the approximation property: there exists a constant $C$ and an integer $k$ such that

$\left\|u-u^{h}\right\|_{H^{1}} \leq C h^{m}\|u\|_{H^{m+1}}, \quad \forall v \in H^{m+1}(\Omega), \quad 1 \leq m \leq k$

where $u^{h}$ is the finite approximation of $u$ and $h$ is the size of the approximation. In the rest of this paper we consider $V^{h}$ like a finite dimensional space with piecewise continuous functions $v^{h} \in C(\bar{\Omega})$ such that for every $K \in \mathcal{T}^{h}, v_{/ K}^{h} \in$ $\mathcal{P}_{1}(K)$, where $\mathcal{T}^{h}$ is a family of element $K$ forming $\Omega$ and $\mathcal{P}_{1}(K)$ is the vectorial space of polynomial of degree less or equal 1 . The family $\left\{V^{h}\right\}$ is a finite approximation of $V$ defined above since if $v_{/ K}^{h} \in \mathcal{P}_{1}(K)$ the trace $\operatorname{Tr}\left(v^{h}\right)$ of $v^{h}$ is a scalar function which belongs to $L_{G}(\Gamma)$.

Now we provide a finite dimensional formulation of the optimality system (4.1) as follows: Find $u^{h}$ and $p^{h}$ such that

$$
\left\{\begin{array}{l}
\int_{\Omega} u^{h} v^{h}+2 \delta t \int_{\Omega} q \nabla u^{h} \nabla v^{h}+2 \delta t \int_{\Gamma_{1}} g\left(u^{h}\right) v^{h}=-\frac{2 \delta t}{\beta} \int_{\Gamma_{2}} p^{h} v^{h}+\int_{\Omega} u^{n+\frac{1}{2}} v^{h} \\
\int_{\Omega} p^{h} \omega^{h}+2 \delta t \int_{\Omega} q \nabla p^{h} \nabla \omega^{h}+2 \delta t \int_{\Gamma_{1}} g^{\prime}\left(u^{h}\right) p^{h} \omega^{h}=2 \delta t \int_{\Omega}\left(u^{h}-u_{o b}^{h}\right) \omega^{h} \\
\sigma^{h}=-\frac{1}{\beta} p^{h}, \quad \text { on } \Gamma_{2}
\end{array}\right.
$$

$$
\begin{aligned}
& \frac{d J}{d \sigma}(\sigma) \zeta=\partial_{1} L(\sigma, u(\sigma)) \zeta-\left\langle p, \partial_{1} F(\sigma, u(\sigma)) \zeta\right\rangle_{Y^{\prime}, Y} \\
& =\beta\langle\sigma, \xi\rangle-\langle p, \mathbf{y}(\xi)\rangle, \\
& \quad \frac{d J}{d \sigma}(\sigma) \zeta=0, \quad \text { for all } \xi
\end{aligned}
$$

is then equivalent to

$\beta\langle\sigma, \xi\rangle_{L^{2}\left(\Gamma_{2}\right)}-\langle p, \mathbf{y}(\xi)\rangle_{L^{2}\left(\Gamma_{2}\right)}=0, \quad$ for all $\xi$
The state problem (4.3) is nonlinear, Newton Raphson numerical method is employed the compute the numerical state solution to this problem which is assembled in GNU Octave-6.1.0 using the usual piecewise finite element approximation. To compute the adjoint state $p^{h}$ in the second equation in the (4.3) LU decomposition is employed to inverse the resulting matrix system. In Figs. 1, 2, 3, 4, 5 and 6 we visualize the solutions of the direct problem (1.1) for 


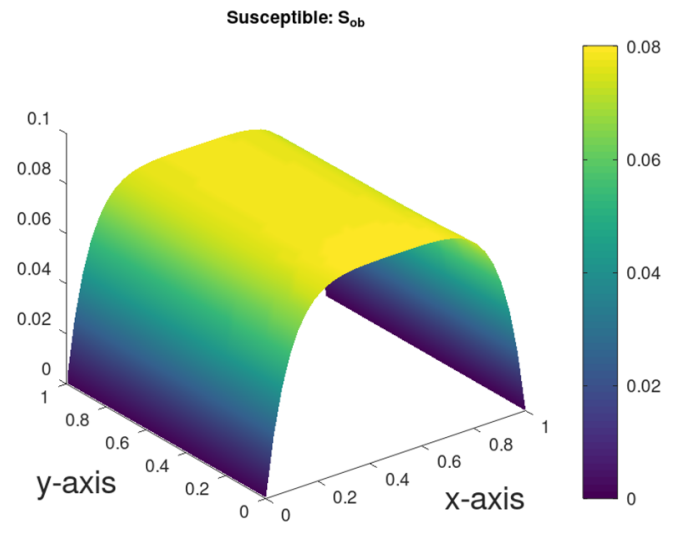

Fig. 1 The diffusion of susceptible individuals $S$ at the second iteration

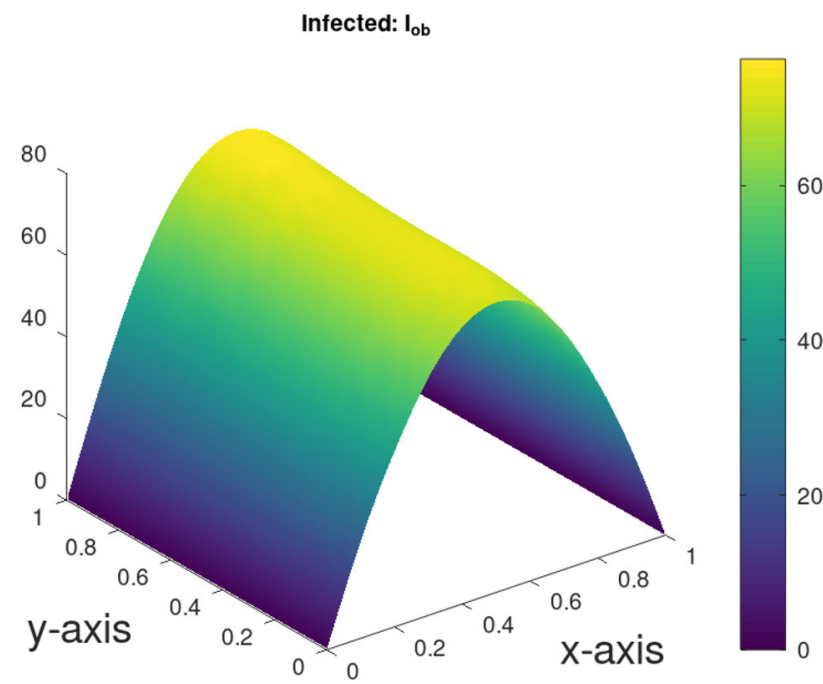

Fig. 2 The diffusion of Infected individuals $I$ at the second iteration

$g=\left[\begin{array}{c}0 \\ \beta_{1} e^{\gamma_{1} I}-\gamma_{1} I-1 \\ 0\end{array}\right], I(0)=1, R(0)=0$, a population of $N=1000$ individuals and the parameter's choice $\gamma=0.7, \beta=0.3, \beta_{1}=0.9$ and $\gamma_{1}=0.4$. Concerning the time discretisation, we take $T=1$ and $\delta t=0.01$ for both problems (2.4)-(2.4). Let $\Omega$ be the unit square defined by $\Omega=(0,1) \times(0,1)$ with boundaries $\Gamma_{1}=[0,1] \times\{0\}$, $\Gamma_{2}=[0,1] \times\{1\}$ and $\Gamma_{3}=\Gamma \backslash\left(\Gamma_{1} \cup \Gamma_{2}\right)$.

\subsection{Example 1}

On the boundary part $\Gamma_{2}$ we define the control $\sigma_{o b}$ as follows:

$\sigma_{o b}=3 \sin (x), \quad x \in \Gamma_{2}$.

In Figs. 1, 2, 3, 4, 5 and 6 we illustrate the solution $(S, I, R)$ with the above choice of parameters $\gamma, \beta, \beta_{1}$ and $\gamma_{1}$ at each time-step $t_{2}$ and $t_{50}$, respectively. At each

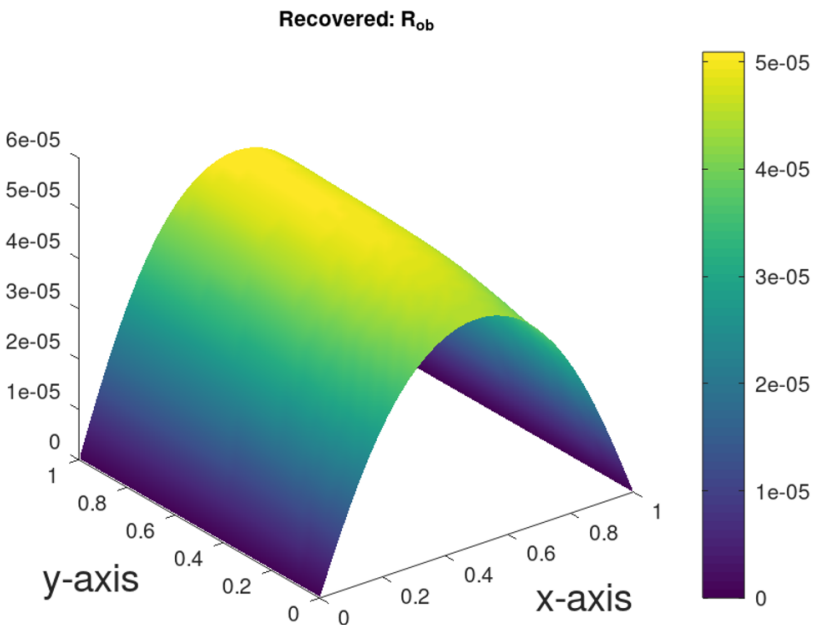

Fig. 3 The diffusion of recovered individuals $R$ at the second iteration

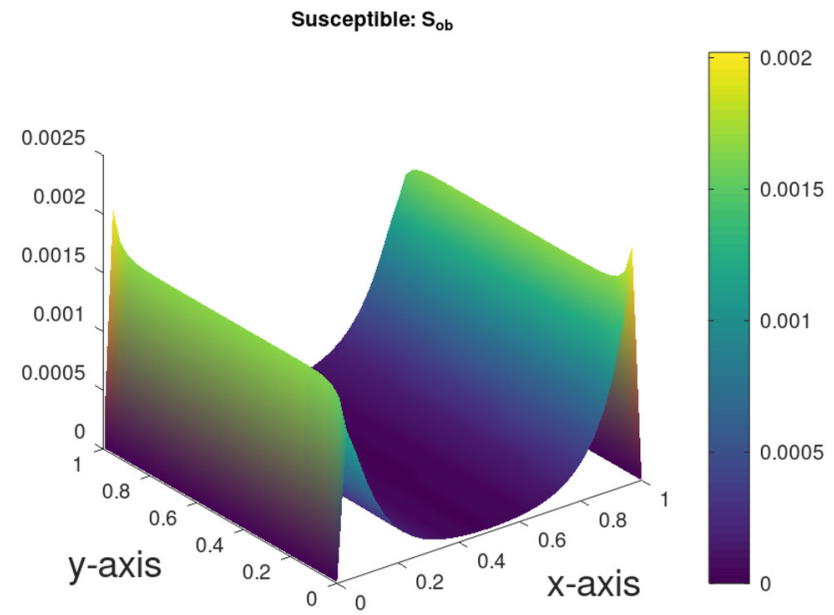

Fig. 4 The diffusion of susceptible individuals $S$ at the iteration 50 . We observe that the number of susceptible diminished regarding the number in the second iteration

time step, the obtained solution presents our observation i.e. $u_{o b}=\left(S, I_{o b}, R\right)$. Figures 7 and 8 show the observation $I_{o b}$ compared with the recovered $I$ at the final time step $T$ and in Fig. 9 we illustrate the given parameter $\sigma_{o b}$ compared with the computed one. Figure 10 shows the observed $I_{o b}$ and the computed $I$ in the zone of the control and we remark that the computed control (optimal control) realises perfectly the desired profile.

\subsection{Example 2}

On the boundary part $\Gamma_{2}$ we define the control $\sigma_{o b}$ as follows:

$\sigma_{o b}=3 \cos (x), \quad x \in \Gamma_{2}$.

In Figs. 11 and 12 we illustrate the computed $I$ and the observed $I$ for this example. In Fig. 13 we visualize the opti- 


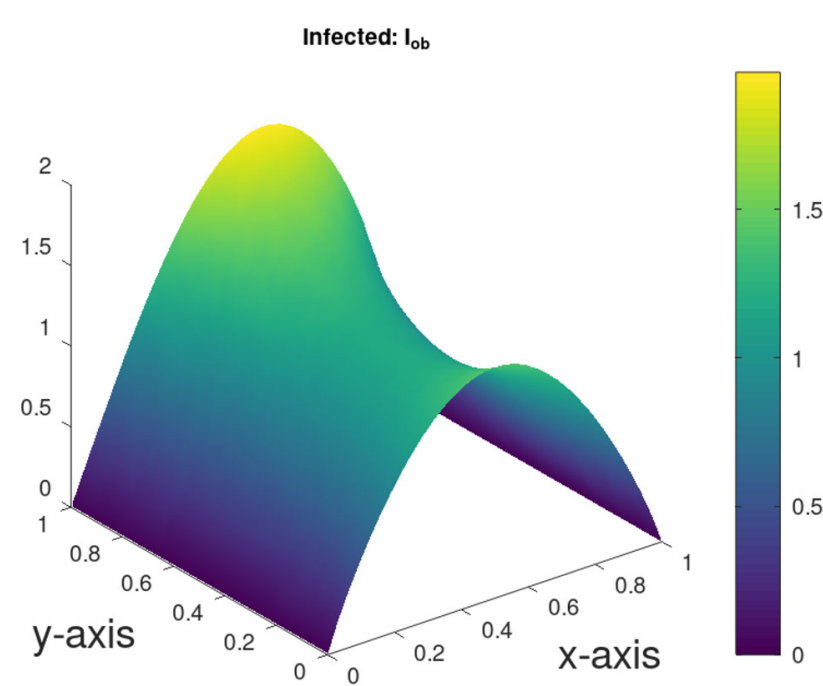

Fig. 5 The diffusion of infected individuals $I$ at the iteration 50

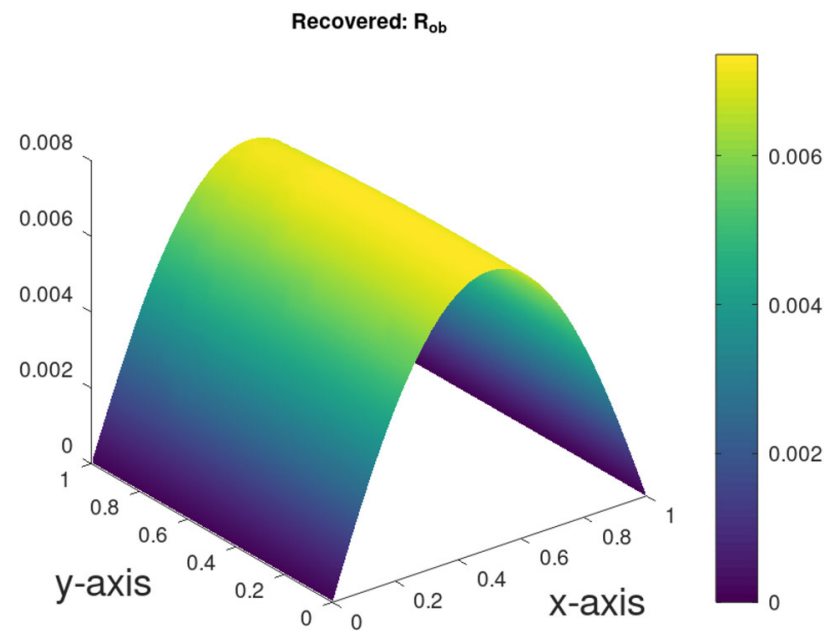

Fig. 6 The diffusion of recovered individuals $R$ at the iteration 50 . Compared with the second iteration, the number of recovered individuals is in augmentation

mal control and the given $\sigma_{o b}$ for the observation. Finally in Fig. 14 we compare the results on the boundary of control.

\section{Conclusion}

In this paper we study a boundary optimal control problem subject to SIR model with nonlinear Robin boundary condition. The existence of the solution of the constraint problem

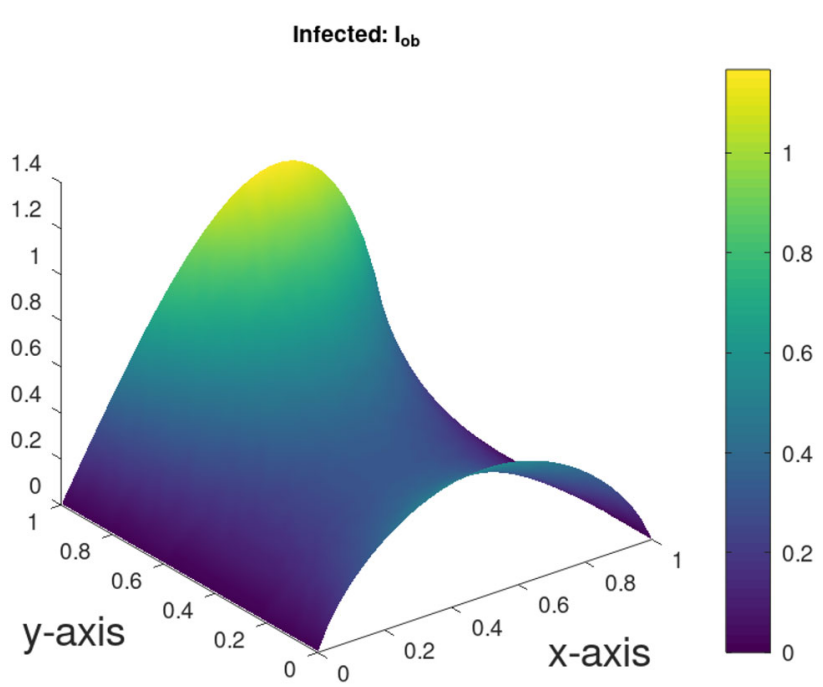

Fig. 7 The computed $I$ at the final time $T$ corresponding to the example 1

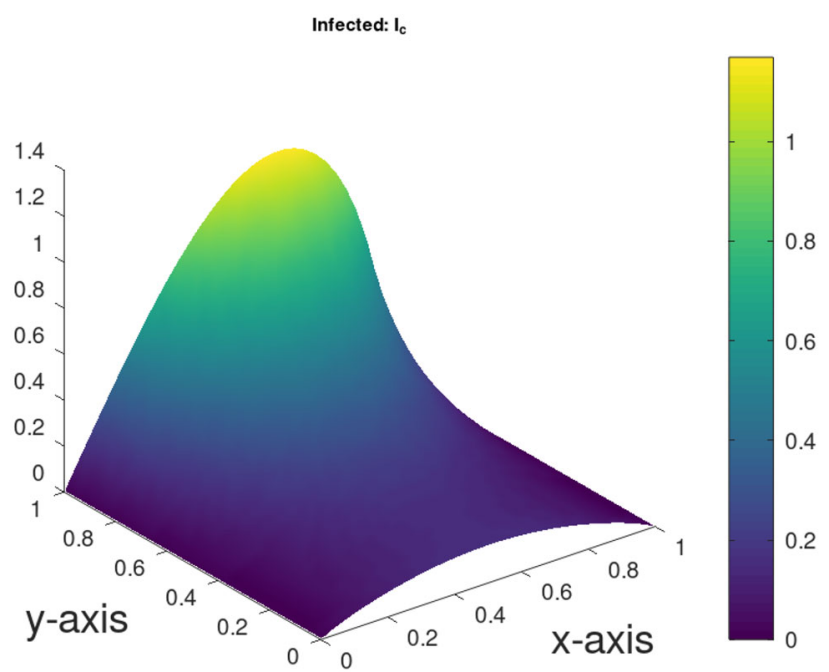

Fig. 8 The observed $I$ at final time $T$ corresponding to the example 1

is obtained in Orlicz space for a specified class of functions defined the part of boundary where the Robin boundary condition is supposed. We provide an optimality condition and a numerical example illustration de solutions. Further-coming works will handle the error estimates corresponding the problem and a possible generalisation to a problem with general nonlinear Robin boundary condition, e.g. nonlinear boundary condition with general function $g$ on the corresponding boundary. 


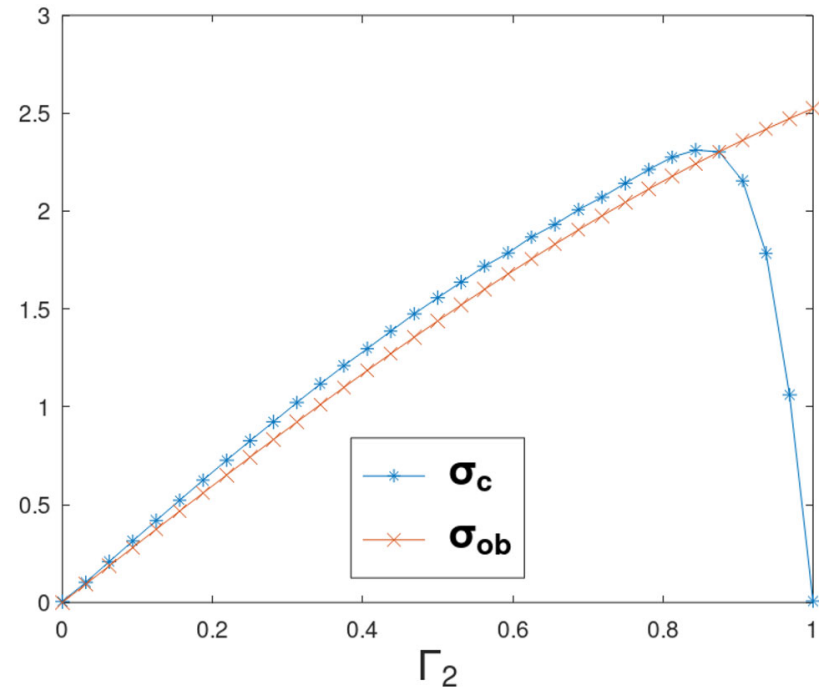

Fig. 9 The exact parameter $\sigma_{o b}$ (in orange x) and the computed optimal control (in blue *). Since we impose the Dirichlet boundary condition in the model we observe that the optimal control vanishes at boundaries $\{0\}$ and $\{1\}$

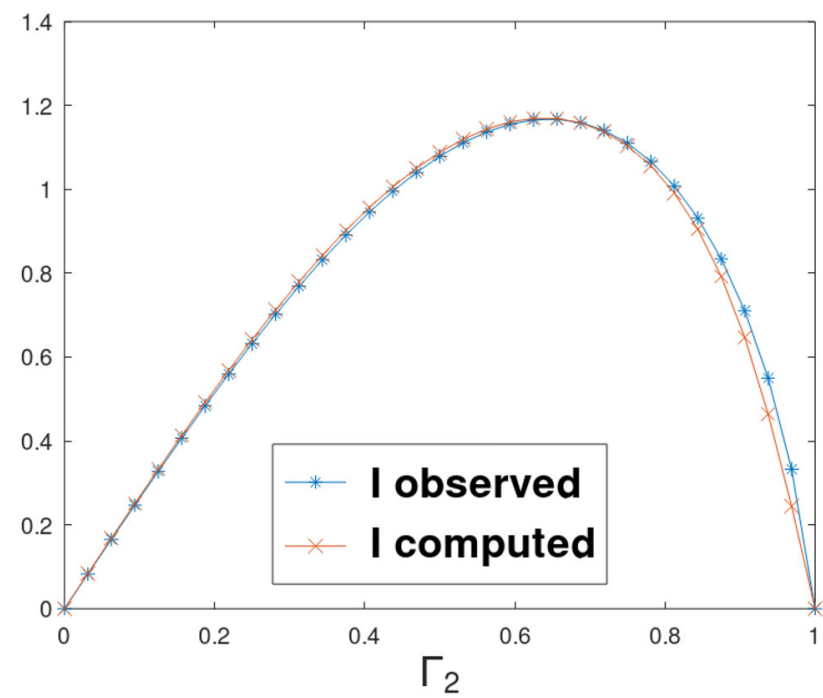

Fig. 10 The exact and the computed $I$ on the boundary of control. We remark that the desired profile is perfectly reached

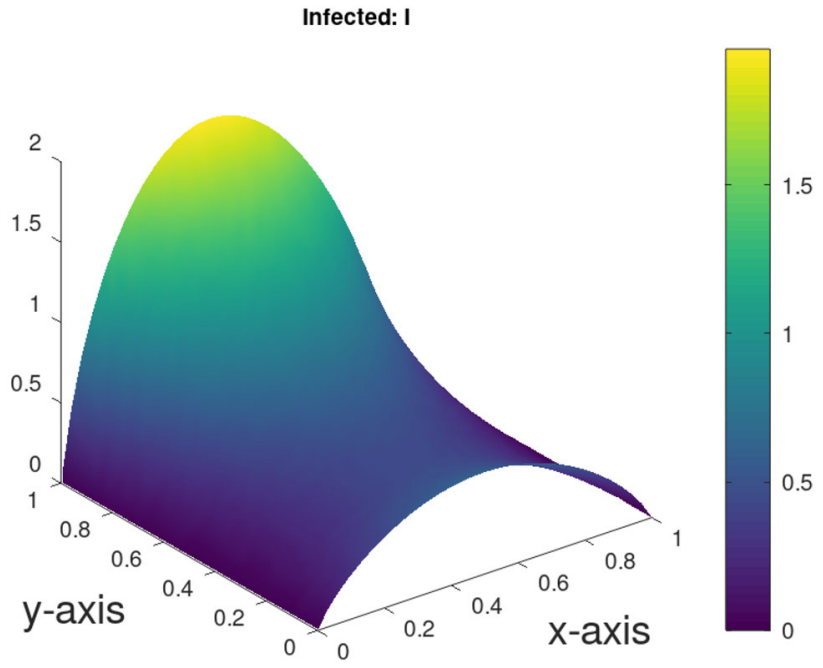

Fig. 11 In this figure, the computed $I$ is visualized at the final time $T$

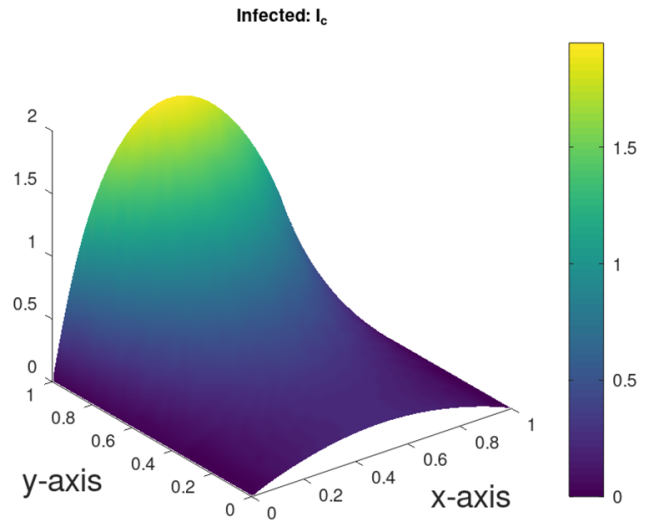

Fig. 12 In this figure we show the exact $I$ at the finel time $T$

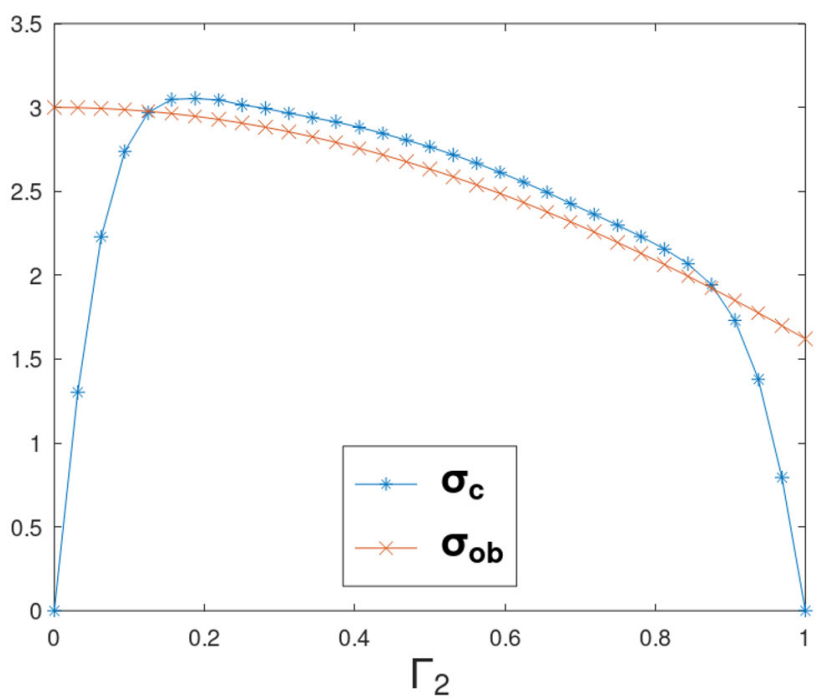

Fig. 13 The exact parameter $\sigma_{o b}$ (in orange x) and the computed optimal control (in blue $*$ ) on the boundary $\Gamma_{2}$. Since we impose the Dirichlet boundary condition in the model we observe that the optimal control vanishes at boundaries $\{0\}$ and $\{1\}$ 


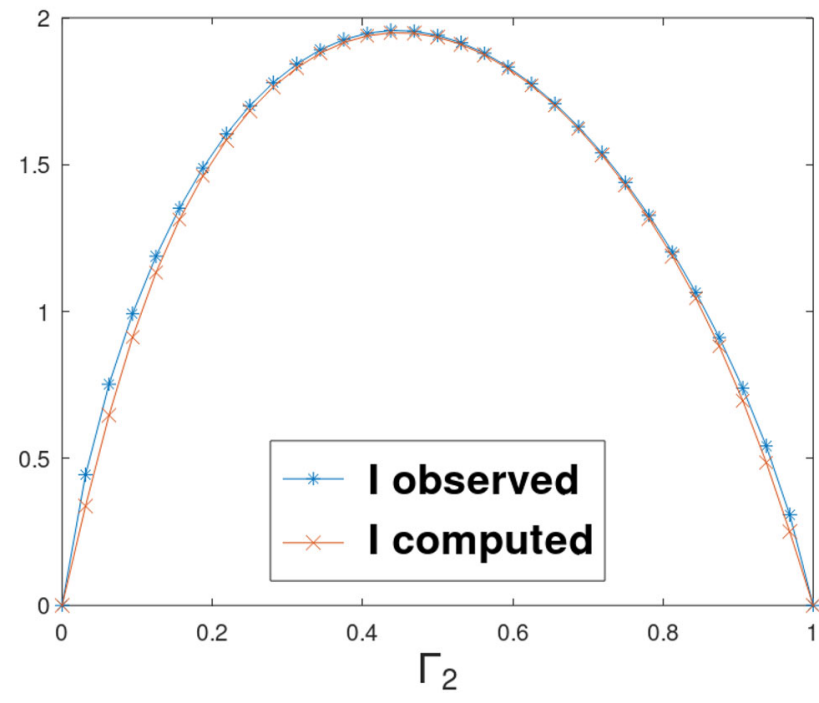

Fig. 14 The exact $I$ (in blue *) and the computed $I$ (in orange $\mathrm{x}$ ) on the boundary of control $\Gamma_{2}$. We remark that the desired profile (number of infected at $\Gamma_{2}$ ) is perfectly reached

Acknowledgements This is supported by CNRST Morocco. The authors of this work would like to express their gratitude to Editors and anonymous Referees for their prolific remarks and valuable suggestions to improve the quality of the paper.

Author Contributions E.-H. Essou and A. Zafrar.

Funding There was no funding.

Data Availability Not applicable.

Code Availability Not applicable.

\section{Declarations}

Conflict of interest I declare that there is no conflict of interest in the publication of this article, and that there is no conflict of interest with any other author or institution for the publication of this article.

Ethical statements I hereby declare that this manuscript is the result of my independent creation under the reviewers' comments. Except for the quoted contents, this manuscript does not contain any research achievements that have been published or written by other individuals or groups. I am the only author of this manuscript. The legal responsibility of this statement shall be borne by me.

\section{References}

1. Brauer F, Castillo-Chavez C, Castillo-Chavez C (2012) Mathematical models in population biology and epidemiology, vol 2. Springer, Berlin

2. Hethcote HW (2000) The mathematics of infectious diseases. SIAM Rev 42(4):599-653

3. Kermack WO, McKendrick AG (1927) A contribution to the mathematical theory of epidemics. Proc R Soc Lond Ser A 115(772):700-721
4. Murray JD (2007) Mathematical biology: I-an introduction, vol 17. Springer, Berlin

5. Brown C (2007) Differential equations: a modeling approach. Sage, Thousand Oaks

6. Keyfitz N (2005) Applied mathematical demography. Springer, Berlin

7. Asano E, Gross LJ, Lenhart S, Real LA (2008) Optimal control of vaccine distribution in a rabies metapopulation model. Math Biosci Eng 5(2):219

8. Lloyd AL, Jansen VAA (2004) Spatiotemporal dynamics of epidemics: synchrony in metapopulation models. Math Biosci 188(12): $1-16$

9. Keeling MJ, Eames KTD (2005) Networks and epidemic models. J R Soc Interface 2(4):295-307

10. Matis JH, Wehrly TE (1994) 17 Compartmental models of ecological and environmental systems. Handb Stat 12:583-613

11. Zakary O, Rachik M, Elmouki I (2017) On the analysis of a multiregions discrete sir epidemic model: an optimal control approach. Int J Dyn Control 5(3):917-930

12. Gatto M, Bertuzzo E, Mari L, Miccoli S, Carraro L, Casagrandi R, Rinaldo A (2020) Spread and dynamics of the covid-19 epidemic in Italy: effects of emergency containment measures. Proc Natl Acad Sci 117(19):10484-10491

13. Giordano G, Blanchini F, Bruno R, Colaneri P, Di Filippo A, Di Matteo A, Colaneri M (2020) Modelling the covid-19 epidemic and implementation of population-wide interventions in Italy. Nat Med 26(6):855-860

14. Hansen E, Day T (2011) Optimal control of epidemics with limited resources. J Math Biol 62(3):423-451

15. Laaroussi AE-A, Rachik M, Elhia M (2018) An optimal control problem for a spatiotemporal sir model. Int J Dyn Control 6(1):384 397

16. Yusuf TT, Benyah F (2012) Optimal control of vaccination and treatment for an sir epidemiological model. World J Model Simul 8(3):194-204

17. Ledzewicz U, Aghaee M, Schättler H (2016) Optimal control for a sir epidemiological model with time-varying populations. In: 2016 IEEE conference on control applications (CCA), IEEE, pp 12681273

18. Cantrell RS, Cosner C (1991) The effects of spatial heterogeneity in population dynamics. J Math Biol 29(4):315-338

19. Cantrell RS, Cosner C (2004) Spatial ecology via reactiondiffusion equations. Wiley, New York

20. Holmes EE, Lewis MA, Banks JE, Veit RR (1994) Partial differential equations in ecology: spatial interactions and population dynamics. Ecology 75(1):17-29

21. Keller JP, Gerardo-Giorda L, Veneziani A (2013) Numerical simulation of a susceptible-exposed-infectious space-continuous model for the spread of rabies in raccoons across a realistic landscape. $\mathbf{J}$ Biol Dyn 7(sup1):31-46

22. Murray J (2003) Spatial models and biomedical applications. Springer, Berlin

23. Wang Q (2019) Qualitative analysis of a Lotka-Volterra predatorprey system with migration. J Math Anal Appl 472(1):421-431

24. Lions JL (1968) Contrôle optimal de systemes gouvernés par des équations aux dérivées partielles. Dunod, Paris

25. Gong W, Yan N (2011) Mixed finite element method for Dirichlet boundary control problem governed by elliptic PDES. SIAM J Control Optim 49(3):984-1014

26. Apel T, Pfefferer J, Rösch A (2015) Finite element error estimates on the boundary with application to optimal control. Math Comput 84(291):33-70

27. Axelsson O, Béreš M, Blaheta R (2021) Computational methods for boundary optimal control and identification problems. Math Comput Simul 189:276-290 
28. Adams RA, Fournier JJF (2003) Sobolev spaces. Elsevier, Amsterdam

29. Hou LS, Turner JC (1995) Analysis and finite element approximation of an optimal control problem in electrochemistry with current density controls. Numer Math 71(3):289-315
30. Amassad A, Chenais D, Fabre C (2002) Optimal control of an elastic contact problem involving tresca friction law. Nonlinear Anal 48(8):1107-1135 